\title{
The Luxated Eddies-Ectopic Meningioma
}

\author{
Anubha Bajaj* \\ Histopathologist in A B Diagnostics, New Delhi, India
}

Submission: March 29, 2021; Published: June 24, 2021

*Corresponding author: Anubha Bajaj, Histopathologist in A B Diagnostics, New Delhi, India

\section{Preface}

Meningioma is a commonly discerned, benign neoplasm of the central nervous system originating from cellular elements of meninges, particularly the arachnoid villous structures and meningothelial cells. The neoplasm is uncommonly delineated within extracranial or ectopic sites. Thus, ectopic meningioma infrequently emerges within extracranial or extra-osseous region of the head and neck or maxilla and mandible and may manifest as a cystoid lesion [1]. Meningioma is appropriately established with ultrastructural examination and cogent immunohistochemistry. The morphology and immunohistochemistry of ectopic meningioma is identical to the frequently discerned intracranial meningioma. Appropriate discernment of an extracranial meningioma is challenging, and misinterpretation may incur an inadequate clinical and surgical management, especially for tumours situated within the facial region.

\section{Disease Characteristics}

Meningioma originates from cellular elements of the meninges such as dura, cap cell layer of arachnoid, arachnoidal granulations, subarachnoid blood vessels, intermingled fibroblasts, and the pia. Majority of lesions adhere to the dura although may infiltrate the bone or originate within an extracranial bone. Commonly, meningioma is adherent to superimposed dura and evolves within the cranial cavity or intra-spinal region [1,2]. The exceptional extracranial meningioma may arise as a common extra-axial neoplasm or appear as a direct extension of a primary intracranial meningioma or may emerge as a true, primary extracranial meningioma engendered from ectopic arachnoid cells [1,2]. Activation of ectopic arachnoid cells or multipotent mesenchymal cells can ensue with diverse surgical procedures such as tooth extraction or chronic inflammation of tooth apices, features which augment proliferation of ectopic cells thus engendering the tumefaction [1]. Ectopic meningioma is frequently misinterpreted, a feature which results in inappropriate therapeutic management. Majority of ectopic meningioma are associated with extended survival and mortality commonly ensues due to adjunctive factors [1,2].

\section{Disease Pathogenesis}

Extracranial meningioma is posited to arise due to diverse mechanisms such as extradural enclosure of arachnoid cell nests during embryogenesis, ectopic cellular migration, development of arachnoid cells in association with peripheral nerves or metaplasia of mature peripheral nerve sheath cells or progenitor cells [2,3]. Ectopic meningioma, un-associated with cranial nerves is postulated to be a mesenchymal neoplasm arising from multipotent mesenchymal stem cells [2,3]. Head and neck tumours are often associated with cranial nerves and are presumed to originate from ectopic arachnoid tissue encompassing the nerves. Therefore, genesis of the tumour can be contemplated to be from perineurial cells of peripheral nerves. Nevertheless, ectopic arachnoid cells within incriminated bone can emerge as a potential source of tumour evolution [2,3].

\section{Clinical Elucidation}

Ectopic meningioma and tumours incriminating the bone may be associated with pain. Enlarged tumefaction situated within a major nerve or abutting a peripheral nerve may be accompanied by concordant signs or symptoms. Facial or extracranial meningioma incriminating the bone is frequently associated with pain, paraesthesia, and anaesthesia. Cogent clinical symptoms are pertinent to anatomic site and anatomic structures adjacent to tumour incrimination $[1,3]$. Ectopic meningioma demonstrates a female predominance with a female to male proportion of 1.2:1 and an estimated $55 \%$ of neoplasms are encountered in females $[2,3]$. Average age of incriminated subjects is 43.4 years, wherein the neoplasm is observed in females at 48.7 years and in males at 36.9 years $[3,4]$. 


\section{Cancer Therapy \& Oncology International Journal}

\section{Histological Elucidation}

Microscopic examination of an incised tissue specimen depicts a tumefaction of mesenchymal origin. Tumefaction is extensively cellular and delineates fascicular, storifom or spindle-shaped cellular pattern. Tumour cells are aligned into cords and whorls $[3,4]$. Meningioma is commonly composed of cellular whorls and rosettes. Tumour cells depict pseudo-nuclear inclusions along with configuration of concentric, calcific concretions denominated as psammoma bodies. Generally, meningioma is composed of spindle-shaped cells which configure whorls, rosettes and interconnecting cellular fascicles. Mitotic figures or cellular and nuclear atypia is usually infrequent. Psammoma bodies may be discerned $[3,4]$. The neoplasm is composed of spindle-shaped cells or sheets of miniature, polygonal cells with prominent nucleoli. Proliferation of spindle-shaped cells and configuration of distinctive whorls is observed. Tumour cells are subdivided by fine collagen bundles. Features of cellular and nuclear atypia such as enhanced cellularity, elevated nuclear to cytoplasmic ratio, tumour necrosis or enhanced mitotic activity are infrequent although may be observed [3,4]. Aggregates of hard, collagenous tissue manifest psammoma bodies. Tumour cells configure reticulin fibres. Tumour tissue may be admixed with fragments of lamellar bone. Foci of bony resorption are observed [3,4] (Figures 1-8).

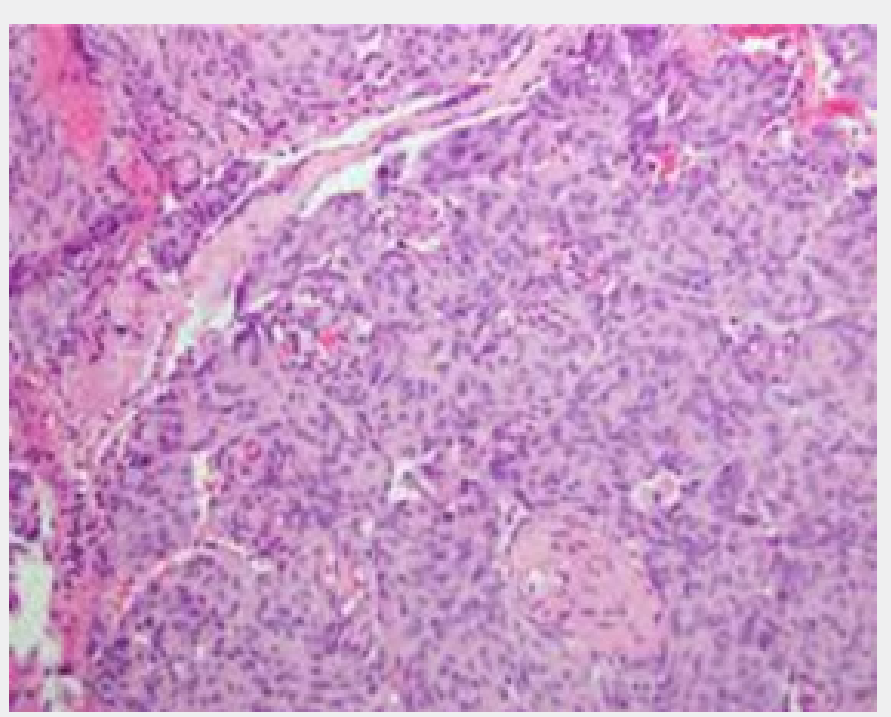

Figure 1: Ectopic meningioma depicting whorls and interlacing fascicles of spindle-shaped cells with minimal atypia [9].

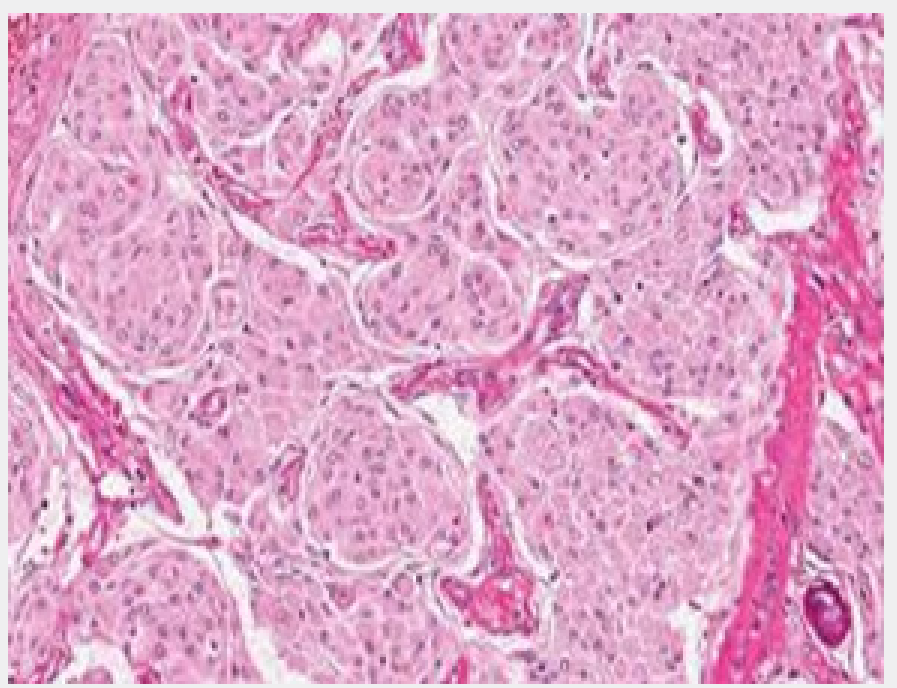

Figure 2: Ectopic meningioma delineating whorls and cellular eddies of spindle-shaped cells divided by fine fibrous tissue septa [10]. 


\section{Cancer Therapy \& Oncology International Journal}

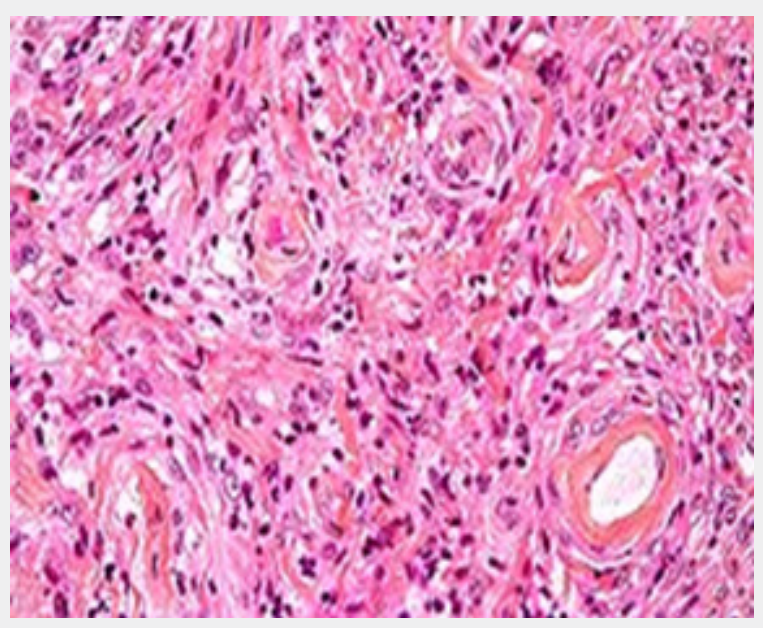

Figure 3: Ectopic meningioma demonstrating spindle-shaped cells with minimal atypia configuring cellular whorls and rosettes admixed with patent vascular articulations [11]

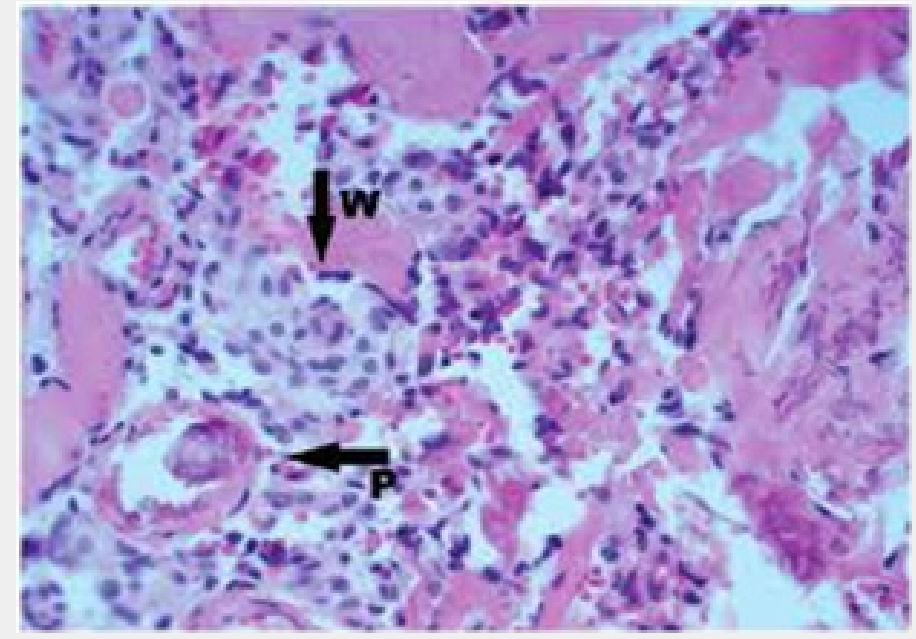

Figure 4: Ectopic meningioma exemplifying whorls of spindle-shaped cells divided by fibrous tissue septa and admixed concentric calcific concretions [12].

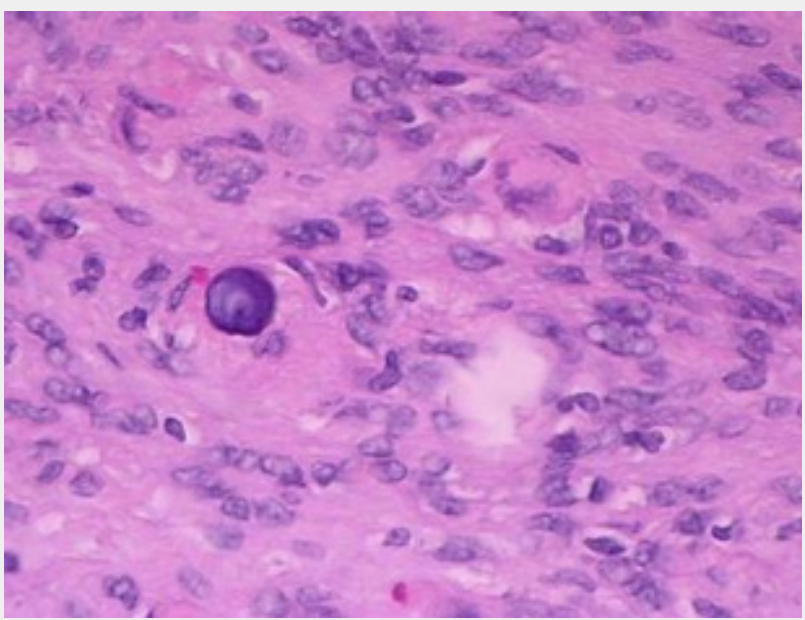

Figure 5: Ectopic meningioma enunciating bundles of spindle-shaped cells configuring whorls and concentric calcific concretions as psammoma bodies [13]. 


\section{Cancer Therapy \& Oncology International Journal}

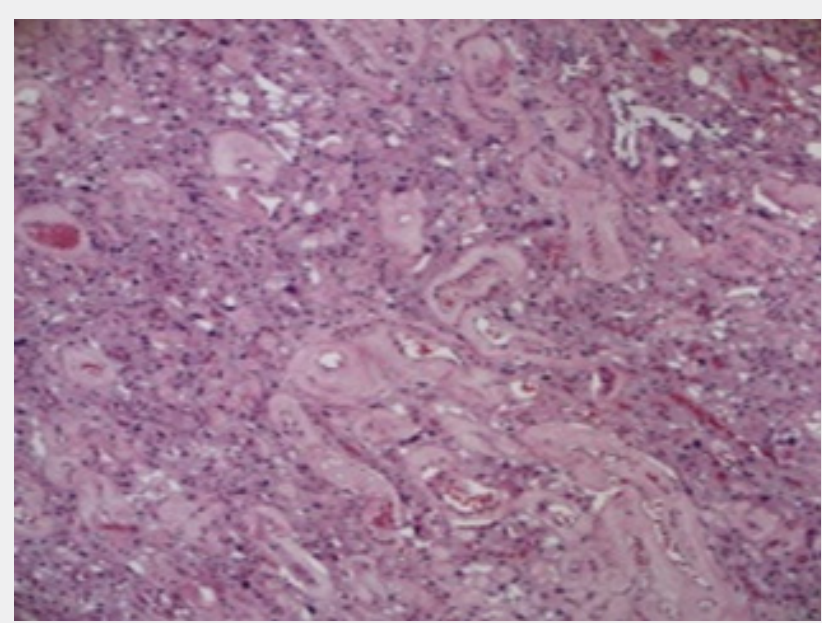

Figure 6: Ectopic meningioma delineating calcific concretions admixed with fascicles of spindle-shaped cells and fine fibrous tissue septa [14].

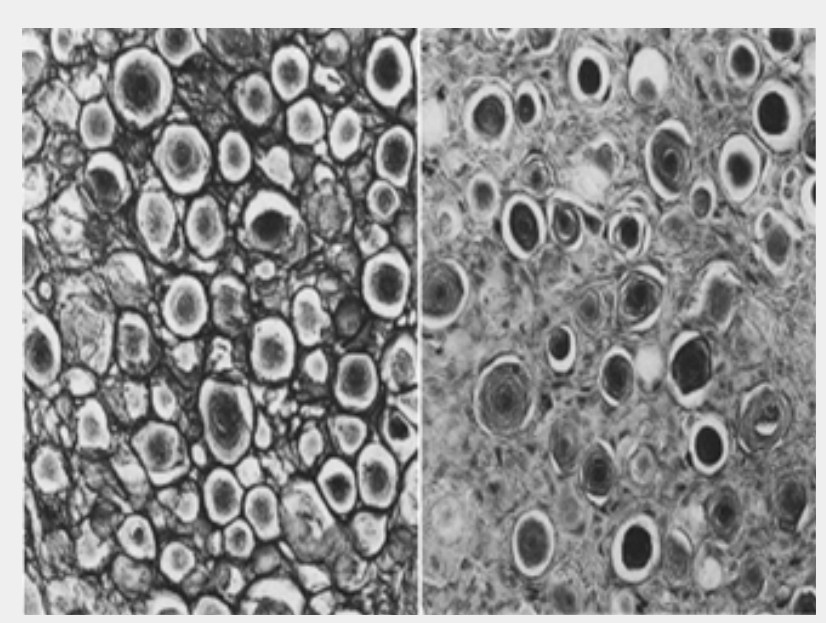

Figure 7: Ectopic meningioma demonstrating fascicles of spindle-shaped cells admixed concentric calcific concretions as the psammoma bodies [15].

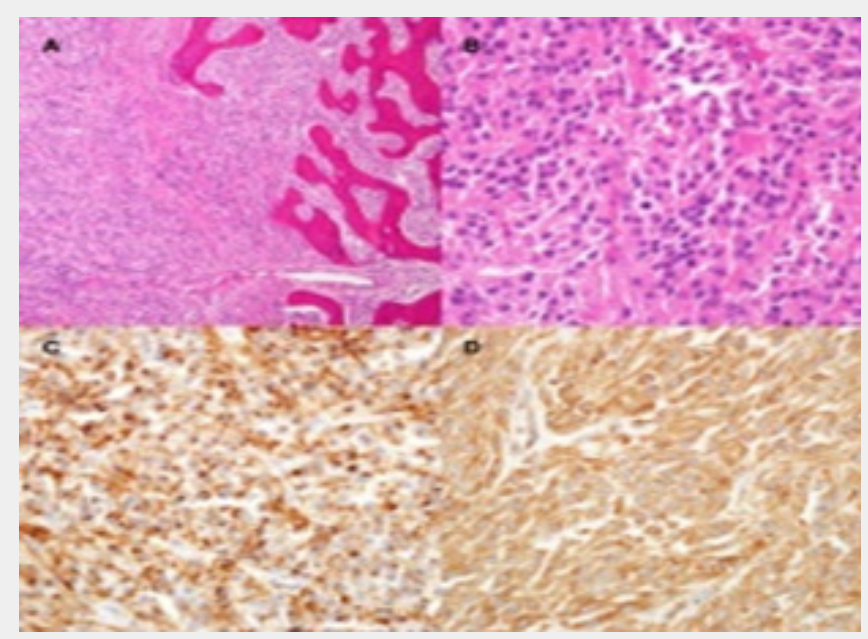

Figure 8: Ectopic meningioma exhibiting whorls of spindle-shaped cells subdivided by fibrous tissue septa and immune reactive epithelial membrane antigen (EMA) and vimentin [16]. 


\section{Cancer Therapy \& Oncology International Journal}

\section{Immune Histochemical Elucidation}

The neoplasm is intensely immune reactive to epithelial membrane antigen (EMA), CD34, vimentin, progesterone receptor and somatostatin receptor 2(SSTR). Few (<20\%) tumours cells are immune reactive to desmoplakin [4,5]. Tumour cells are immune non-reactive to actin, desmin, cytokeratin AE1/AE3, CK18, smooth muscle actin (SMA), carcinoembryonic antigen (CEA), anti-melanoma antibodies, claudin-1, S100 protein and glucose transporter-1 (glut-1). Additionally, a decimated Ki-67 proliferation index $(<5 \%)$ is associated with an indolent clinical course $[4,5]$.

\section{Differential Diagnosis}

Ectopic meningioma mandates a segregation from diverse neoplasms of peripheral nerve origin which require a cogent immunohistochemistry to appropriately demonstrate epithelial and mesenchymal segments of tumour cells $[5,6]$. Ectopic meningioma requires a distinction from perineurioma, schwannoma, multi-locular ameloblastoma, odontogenic keratocyst, neurofibroma or paraganglioma $[5,6]$. perineurioma is an exceptional lesion which simulates meningioma. Perineurial cells represent the peripheral counterpart of meningeal cells, a feature which engenders identical morphology of the lesions. An adequate clinical distinction is also mandated as a perineurioma demonstrates limited possible infiltration of abutting anatomical structures and is associated with minimal morbidity. Perineurioma depicts minimal localized tumour reoccurrence and can be managed with minimalistic surgical intervention. Besides, adjunctive radiation therapy may not be mandated for treating infiltrative perineurioma. As perineurioma morphologically simulates a meningioma, immunohistochemistry is pertinent in demarcating the conditions. Perineurioma is uniformly immune reactive to epithelial membrane antigen (EMA) and intensely immune reactive to claudin-1. Immune reactive glut- 1 defines malignant potential and infiltrative capacity of the neoplasm. Perineurioma is immune non-reactive to S100 protein, CD34 and smooth muscle actin (SMA) $[5,6]$.

Multi-locular ameloblastoma is a neoplasm depicting islands of odontogenic epithelium with peripheral palisading, reverse polarization, stellate- reticulum like cells and a supra-basal layer of loosely arranged, angulated cells. Dentin or enamel is usually absent [5,6]. odontogenic keratocyst denominates a cystic cavity layered with uniform, six to eight cell thick epithelium with palisaded, hyperchromatic nuclei and epithelial buds arising from the basal layer. Luminal surface depicts corrugated, parakeratotic cells. Artefactual clefts appear between the epithelium and underlying fibro-connective tissue [5,6]. Neural lesion as a schwannoma is composed of hyper-cellular Antoni A areas admixed with hypo-cellular Antoni B areas. Nuclear palisading around fibrillary processes appears as Verocay bodies, denominated within hyper-cellular areas along with enlarged vascular articulations [6]. Tumour cells are elongated, wavy and tapered with ill-defined cytoplasm and dense nuclear chromatin. Mitosis is exceptional. Tumefaction is immune reactive to S100 protein and immune non-reactive to epithelial membrane antigen (EMA) [5,6]. neurofibroma is composed of proliferation of cellular components of a peripheral nerve with discernible Schwan cells, mast cells, axons, fibroblasts, Wagner- Meissner corpuscles, Pacinian corpuscles, stromal muco-substances, and collagen. The neoplasm is immune reactive to $\mathrm{S} 100$ protein, CD34, SOX10, Factor XIIIa, calretinin, neurofilaments and collagen type IV. Tumefaction is immune non-reactive to epithelial membrane antigen (EMA), desmin, cytokeratin and smooth muscle antigen (SMA) $[5,6]$.

\section{Investigative Assay}

Extracranial meningioma characteristically displays a well-defined, osteolytic or a mixed, osteolytic-osteosclerotic, radiolucent lesion associated with cortical displacement, thinning and eventual disruption of cortical bone. Bone lesions frequently depict incrimination of peripheral nerve. Interruption of cortical bone margins are indicative of aggressive biological behaviour $[7,8]$. Extensive, panoramic plain radiographs can aptly visualize the lesion and demonstrate a poorly circumscribed, multi-locular, osteolytic lesion within the incriminated bone $[7,8]$. Cone beam computerized tomography (CBCT) is adopted to determine tumour extension and implication of adjacent anatomic structures. Hypodense, osteolytic lesions with ill-defined boundaries and rupture of adjacent bone is observed. Tumefaction may infiltrate the Haversian canal system. Cellular whorls or pseudo-onion bulbs are reminiscent of perineurioma or meningioma $[7,8]$. Osteolytic or admixed radiolucent-radiopaque tumefaction arising within the mandible or facial bones rarely appear because of primary tumours of the central nervous system and may arise on account of neural cell neoplasms. Upon panoramic radiographic assessment and computed tomography (CT), an expansive, radiolucent lesion of variable magnitude is discerned which penetrates the bone cortex $[7,8]$. Tumefaction may be devoid of distinctive clinical symptoms or typical radiographic features upon panoramic radiography or three- dimensional (3D) imaging. However, computerized tomography (CT) can significantly assess concurrence of the neoplasm, incriminated bone and exclude possible malignant transformation $[7,8]$.

\section{Therapeutic Options}

Surgical intervention with partial excision of incriminated bone and soft tissue along with reconstruction of surgical defect employing an autologous bone graft is optimal and can be suitably adopted $[7,8]$. Comprehensive surgical eradication of the tumefaction is mandated to circumvent localized tumour reoccurrence. Preferred therapy for an extracranial meningioma is competent surgical extermination of the neoplasm $[7,8]$. Postoperative panoramic radiograph may demonstrate an adequate positioning of the graft with bone graft repair. Subsequently, subjects remain disease free $[7,8]$. Extensive followup of ectopic meningioma demonstrates a lack of localized tumour 


\section{Cancer Therapy \& Oncology International Journal}

reoccurrence. Extensive tumour monitoring is recommended, especially in instances depicting cytological atypia.

Prognostic outcomes of extracranial meningioma treated with comprehensive surgical eradication is favourable and is accompanied by disease-free percentage of $82 \%$ at 5 years and $78 \%$ at 10 years $[7,8]$. Instances subjected to subtotal tumour resection or aggressive tumefaction demonstrating morphological features such as mitotic activity or foci of necrosis necessitate extensive tumour monitoring. However, certain neoplasms with follow-up at 12 months with the employment of panoramic radiographs and computerized tomographic (CT) imaging can depict an absence of tumour progression or additional clinical symptoms. Aforesaid features indicate a lack of requirement of mandatory, immediate surgical intervention $[7,8]$.

\section{References}

1. Shuangshoti S, Panyathanya R (1973) Ectopic meningiomas. Arch Otolaryngol 98(2): 102-105.

2. Rommei N, Bissinger O, Andrea Rau, Thomas Muecke (2017) Ectopic meningioma of the mandible in a 20-year old woman- a case report and literature review. J Surg Case Rep 2017(3): rjx 047.

3. Inara Carneiro Costa Rege, Robson Rodrigues Garcia, Elismauro Francisco Mendonca (2017) Primary extracranial meningioma- a rare location. Head Neck Pathol 11(4): 561-566.

4. Iaconetta G, Santella A, Marco Friscia, Vincenzo Abbate, Luigi Califano
(2012) Extracranial primary and secondary meningiomas. Int J Oral Maxillofac Surg 41(2): 211-217.

5. Rushing EJ, Bouffard JP, Sherman McCall, Cara Olsen, Hernando Mena, et al. (2009) Primary extracranial menigiomas: an analysis of 146 cases. Head Neck Pathol 3(2): 116-130.

6. Lell M, Tudor C, Aigner T, P Kessler (2007) Primary intraosseous meningioma of the mandible-CT and MR imaging features. AJNR Am J Neuroradiol 28(1): 129-131.

7. Mosqueda-Taylor A, Dominguez-Malagon H, Ana-Maria CanoValdez, Ana-Maria Montiel-Hernandez (2009) Primary extracranial meningioma of the mandible. Med Oral Patol Oral Cir Bucal 14(4): E167-E170.

8. Possanzini P, Pipolo C, S Romagnoli, M Falleni, L Moneghini, et al (2012) Primary extra-cranial meningioma of head and neck: clinical, histopathological and immunohistochemical study of three cases. Acta Otorhinolaryngol Ital 32(5): 336-338.

9. Image 1 Courtesy: Clinics in surgery.

10. Image 2 Courtesy: Sage journals.

11. Image 3 Courtesy: Wikipedia

12. Image 4 Courtesy: Tandofline.com

13. Image 5 Courtesy: Medscape reference

14. Image 6 Courtesy: Pathology outlines.

15. Image 7 Courtesy: Journal of neurosurgery.com

16. Image 8 Courtesy: BMC Cancer.
This work is licensed under Creative Commons Attribution 4.0 License DOI:10.19080/CTOIJ.2021.19.556007 